\title{
MAGNETIC DIPOLE INFLATION WITH CASCADED ARC AND APPLICATIONS TO MINI-MAGNETOSPHERIC PLASMA PROPULSION \\ L. Giersch", R. Winglee ${ }^{\dagger}$, J. Slough ${ }^{\ddagger}$, T. Ziemba ${ }^{\S}$, P. Euripides", University of Washington, Seattle, WA, 98195
}

\begin{abstract}
Mini-Magnetospheric Plasma Propulsion (M2P2) seeks to create a plasma-inflated magnetic bubble capable of intercepting significant thrust from the solar wind for the purposes of high speed, high efficiency spacecraft propulsion. Previous laboratory experiments into the M2P2 concept have primarily used helicon plasma sources to inflate the dipole magnetic field. The work presented here uses an alternative plasma source, the cascaded arc, in a geometry similar to that used in previous helicon experiments. Time resolved measurements of the equatorial plasma density have been conducted and the results are discussed. The equatorial plasma density transitions from an initially asymmetric configuration early in the shot to a quasisymmetric configuration during plasma production, and then returns to an asymmetric configuration when the source is shut off. The exact reasons for these changes in configuration are unknown, but convection of the loaded flux tube is suspected. The diffusion time was found to be an order of magnitude longer than the Bohm diffusion time for the period of time after the plasma source was shut off. The data collected indicate the plasma has an electron temperature of approximately $11 \mathrm{eV}$, an order of magnitude hotter than plasmas generated by cascaded arcs operating under different conditions. In addition, indirect evidence suggests that the plasma has a $\beta$ of order unity in the source region.
\end{abstract}

\section{INTRODUCTION}

The solar wind is a high velocity $<10^{5}$ $\mathrm{m} / \mathrm{s}$ ) plasma with $\sim 2 \mathrm{nPa}$ of dynamic pressure. In order to intercept $\sim 1 \mathrm{~N}$ of thrust from this pressure, a surface $\sim 18 \mathrm{~km}$ in radius is required. The required magnetic field at this surface must have a magnetic pressure of $1 \mathrm{nPa}$, implying a filed strength of $\sim 35 \mathrm{nT}$. Obtaining this magnetic field at $18 \mathrm{~km}$ from a vacuum dipole magnet (B scales with $\left.1 / r^{3}\right)$ is highly impractical.

\footnotetext{
* Graduate Student, Aeronautics and Astronautics

† Professor, Aeronautics and Astronautics

$\mp$ Professor, Aeronautics and Astronautics

$\S$ Graduate Student, Aeronautics and Astronautics

II Postdoc, Earth and Space Sciences
}

As an alternative, Mini-Magnetospheric Plasma Propulsion (M2P2) seeks to create a plasma-inflated magnetic bubble capable of intercepting significant thrust from the solar wind. One of the critical factors for plasma-inflation is the creation of high $\beta$ plasma. $\beta$ is the ratio of plasma pressure (proportional to density $\times$ temperature) to magnetic field pressure (proportional to the square of the magnetic field strength). In theory, a high $\beta$ plasma with a large Magnetic Reynolds number injected into a modest $(\sim 1 \mathrm{~m}, 0.1 \mathrm{~T})$ magnetic dipole will carry the magnetic field outward ("inflate") as the plasma expands. The condition of a large Magnetic Reynolds number indicates that the plasma pushes the magnetic field outward faster than the magnetic field can diffuse inward through the plasma. The resulting magnetic field scales as a current sheet (B scales with $1 / r$ ), and it then becomes possible to produce the required magnetic field at the required distances to yield thrusts $\sim 1 \mathrm{~N}$. The M2P2 system can thus be used as a high $\Delta V$, high efficiency propulsion system for a modest $(100 \mathrm{~s}$ of $\mathrm{kg}$ ) interplanetary spacecraft, using reasonable amounts of onboard power (few 10 s of $\mathrm{kW}$ and consumables.

The M2P2 system is more akin to a solar sail than a traditional electric thruster. However, a solar sail uses a material boundary to intercept an ambient energy flux (solar photons), and the issues of material development and structural deployment must be addressed. The M2P2 uses an electro-dynamic boundary to intercept the solar wind, eliminating these issues.

Previous laboratory experiments into the M2P2 concept have used helicon plasma sources to inflate the dipole magnetic field ${ }^{2}$. These sources use radio waves to generate and heat a neutral gas to plasma conditions. Cascaded arcs have also been used in M2P2 laboratory investigations, albeit to a lesser extent ${ }^{3}$. The work presented here further expands the investigation of the cascaded arc as a plasma source for M2P2 experiments. The cascaded arc works by drawing electrical current through plasma located between the electrodes of the source. This ohmically heats the plasma until it escapes the source via a central hole in the anode. The cascaded arc can thus deposit $100 \mathrm{~kW}$ into the plasma, orders of

1

American Institute of Aeronautics and Astronautics 
magnitude more than the helicon source used previously.

\section{PLASMA SOURCE}

The cascaded arc source used here was designed to be placed inside an electromagnet and flow plasma from the interior region of the magnet to the exterior region along the magnetic field lines. A typical (single ended) cascaded arc consists of three basic components in a cylindrical geometry. A stack of conducting plates that are electrically insulated from each other and the electrodes separate an anode and cathode. A hole drilled through the electrodes and plates is filled with gas, and an electrical current passed between the electrodes ohmically heats the gas to plasma conditions. It was desired to have the plasma flow from both the "top" and "bottom" of the source simultaneously, and so a "double ended" cascaded arc was developed (Figure 1). This source had two anodes (one top, one bottom) and a single central cathode. Two separate capacitor banks held the voltage from the individual anodes relative to the cathode. Separate current limiting resistors placed between the positive capacitor terminals and the anodes regulated the maximum current that could flow through the circuit. The current return from the common cathode passed through a single IGBT before returning to the common negative terminal of the capacitor banks. This allowed the single IGBT to gate the current through both ends of the source. The anode voltages relative to the cathode were measured, as was the voltage drop across the current limiting resistors. This provided a simple way of measuring the current passing through the plasma as well as the ohmic power input. Neon gas entered the source through a $1 / 4^{n}$ diameter hole in the cathode connected via tubing to a gas puff valve outside the vacuum chamber.

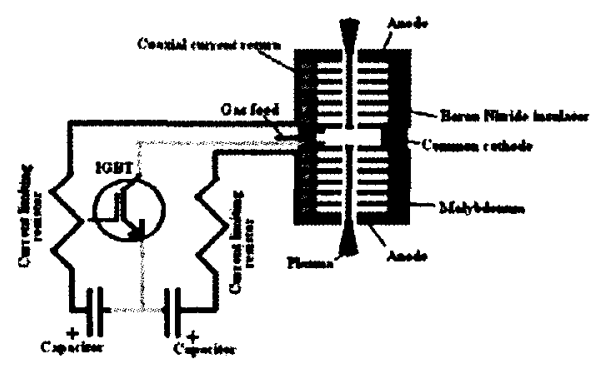

Figure 1. Diagram of the double-ended cascaded arc plasma source

\section{EXPERIMENTAL SETUP}

The double-ended cascaded arc plasma source was placed inside a $13.5 \mathrm{~cm}$ radius, $0.04 \mathrm{~T}$ dipole electromagnet. This configuration of placing the plasma source inside the dipole magnet is similar to those used in previous helicon ${ }^{2}$ and cascaded $\operatorname{arc}^{3} \mathrm{M} 2 \mathrm{P} 2$ experiments. The electrical feeds of the dipole magnet and arc source, the support structure, and the gas feed for the source were brought out along the axis of the magnet in order to minimize the plasma losses to any physical boundaries. The dipole and plasma source system were then placed in a $1 \mathrm{~m}$ diameter, $0.6 \mathrm{~m}$ tall vacuum chamber (Figure 2 ).

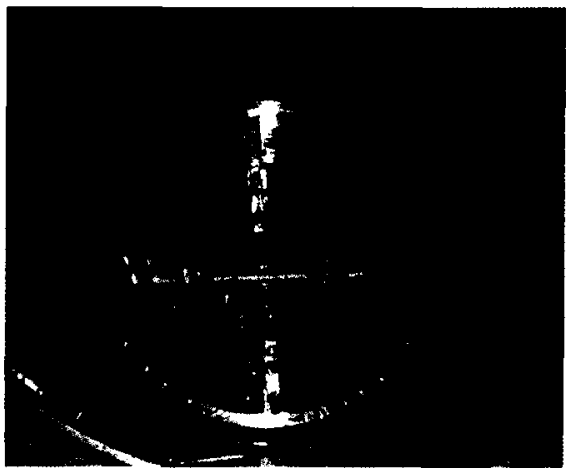

Figure 2. Photo of the dipole and plasma source system inside the vacuum chamber

The chamber was wrapped with a set of approximately Helmholtz coils to provide an optional, DC external magnetic field of roughly $0.005 T$. When used, the external field forced the dipole field lines in from the vacuum chamber wall and imposed a separatrix on the magnetic field configuration (Figure 3).
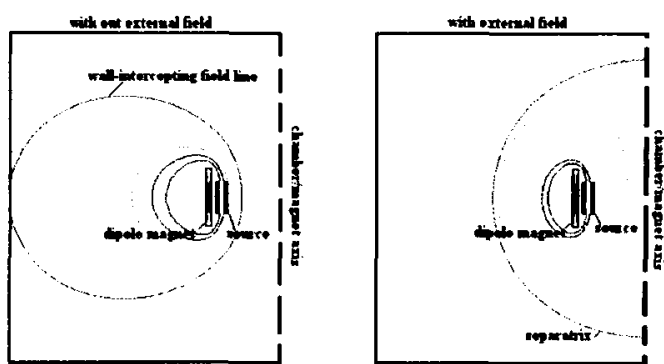

Figure 3. Magnetic field lines of the dipole with and without the external field

\section{RESULTS}

Photographs of the experiment during operation were obtained using a digital camera placed inside the vacuum chamber (Figure 4 ). The

American Institute of Aeronautics and Astronautics 
field of view is limited by the size of the chamber. Under normal operating conditions, plasma is only visible near the top and bottom of the plasma source (located inside the magnet). To view the plasma elsewhere, the vacuum chamber must be backfilled with neutral neon gas prior to operation. Under these conditions, the plasma is clearly seen to flow along the magnetic field lines. Furthermore, activating the external magnetic field clearly changes the magnetic field geometry and consequently forces the plasma closer to the magnet. These conclusions are supported by plasma density measurements discussed shortly.
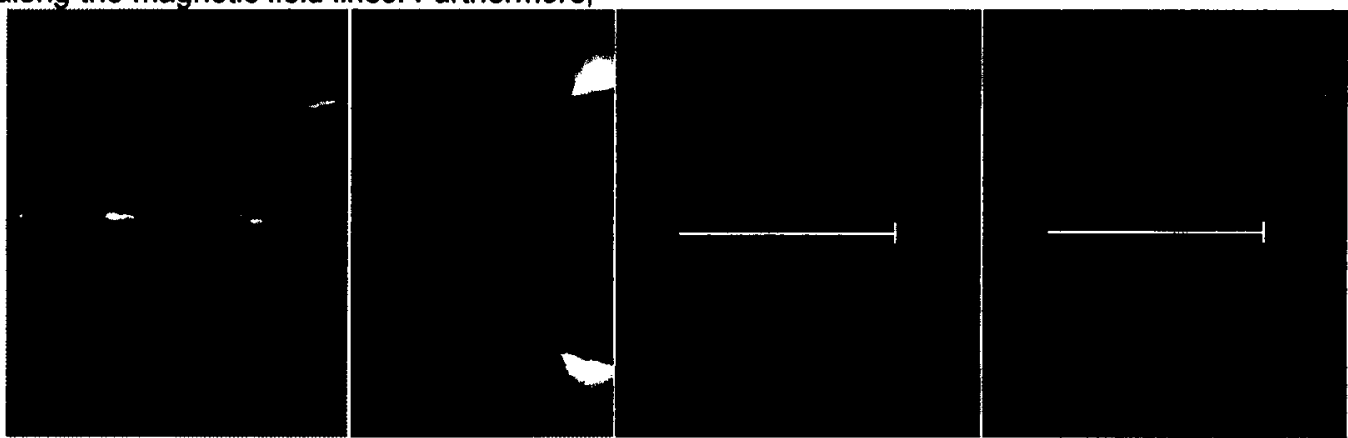

Figure 4. From left to right, the magnet, the plasma under normal operating conditions, the plasma with backfill, and the plasma with backfill and external field. While some photos are cropped, all are on the same scale and taken from the same vantage point. The white bars in the backfill pictures are the same size and are provided as an aid to scale.

The voltage across and current through both ends of the arc were measured, and from these the total power into the arc was determined (Figure 5). The arc was activated for $500 \mu \mathrm{sec}$, depositing an approximate average of $100 \mathrm{~kW}$ into the plasma during that time. Each end of the arc drew an approximate average of 400 Amps during that time.

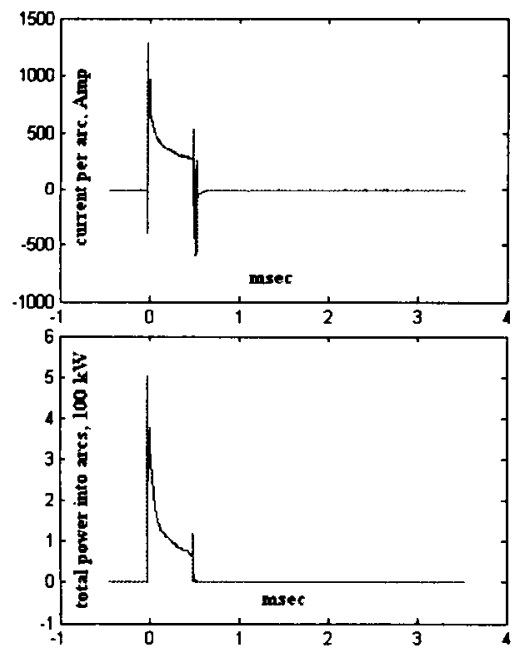

Figure 5. Current and power through the arc vs. time

\section{Electron Temperature}

Double Langmuir probes were arranged at $45^{\circ}$ increments along the vacuum vessel.

Sweeping the voltage across the Langmuir probes over multiple shots yielded the electron temperature of the plasma (Figure 6). In this way the electron temperature was measured at several radial and azmuthal locations and at different times during the shot, showing no significant variation beyond the error bars of the measurement (Table 1). These measurements were done both with and without the external field, and showed no significant change beyond the error bars of the measurement. The average electron temperature is $11.6 \mathrm{eV}$, an order of magnitude hotter than the electron temperatures typically found in other, lower power cascaded arcs 4 .

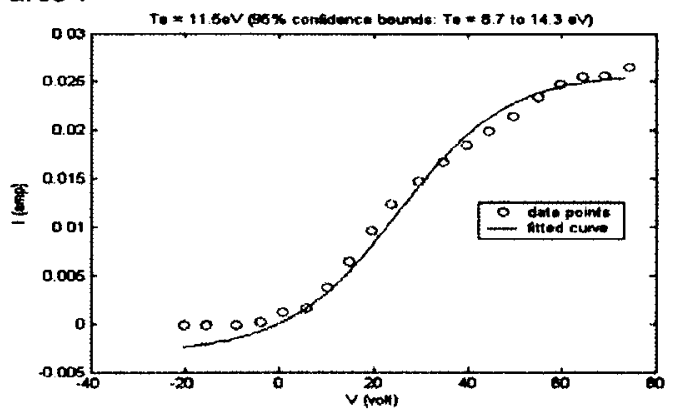

Figure 6. Langmuir characteristic curve 


\begin{tabular}{|l|l|l|}
\hline $\begin{array}{l}\text { Probe } \\
\text { Location }\end{array}$ & $\begin{array}{l}\text { Temp } \\
\mathrm{eV}\end{array}$ & $\begin{array}{l}95 \% \text { bounds } \\
\mathrm{eV}\end{array}$ \\
\hline $\mathrm{R}=25.25 \mathrm{~cm}$, Front & 14 & $11.1-16.9$ \\
\hline $\mathrm{R}=22.25 \mathrm{~cm}$, Front & 11 & $9-13$ \\
\hline $\mathrm{R}=19.25 \mathrm{~cm}$, Front & 11.5 & $8.7-14.3$ \\
\hline $\mathrm{R}=16.25 \mathrm{~cm}$, Front & 11.9 & $8.2-15.6$ \\
\hline $\mathrm{R}=21.25,90^{\circ}$ & 13.4 & $10.6-16.3$ \\
\hline $\begin{array}{l}\mathrm{R}=21.25,90^{\circ}, \\
\text { Toff }+225 \mu \mathrm{sec}\end{array}$ & 11 & $7.4-14.7$ \\
\hline $\begin{array}{l}\mathrm{R}=25.25,90^{\circ}, \\
\text { Ext. field on }\end{array}$ & 8 & $4.5-14$ \\
\hline $\begin{array}{l}\mathrm{R}=17.25 \mathrm{~cm}, \\
\text { Ext. field on }\end{array}$ & 12 & $8.3-14.6$ \\
\hline
\end{tabular}

Table1: Electron temperatures

Assuming the electrons carried the current in the arc ( $\sim 400 \mathrm{Amps})$ at the thermal velocity found using the swept Langmuir probes described above $\left(11.6 \mathrm{eV} \rightarrow \approx 2 \times 10^{6} \mathrm{~m} / \mathrm{s}\right)$, the electron density in the source is approximated by $n \approx 4 \times 10^{19} \mathrm{~m}^{-3}$. This assumes that the plasma is uniformly distributed evenly across the entire plasma source bore $\left(3.2 \times 10^{-5} \mathrm{~m}\right)$, making this a lower bound on the maximum density. Indeed, if the plasma column has a radius $1 / 3$ that of the bore, the density jumps by roughly an order of magnitude. If this is the case, the local $\beta$ of the plasma at the source is on the order of 1 . Densities greater than $10^{21} \mathrm{~m}^{-3}$ have been measured in cascaded arcs passing 50 Amps of current ${ }^{4}$. This $\beta \sim 1$ in the source region may account for the lower than expected equatorial densities measured, as will be discussed next.

\section{EQUATORIAL PLASMA DENSITY}

During multiple shots, the probes were radially repositioned, providing a $2 \mathrm{D}$ (radialazmuthal) map of the plasma density in the equator of the magnet vs. time. This map was done both with and without the external magnetic field. In both cases, the plasma density exhibits a radial peak. The location of the radial peak depends on whether or not the external field is applied (Figure 7). It is seen that the radial peak location is closer to the dipole magnet when the external field is applied. This correlates to the field lines that map through the plasma source also moving in closer to the dipole wall when the external field is applied. This is believed to indicate that the equatorial plasma is well magnetized.
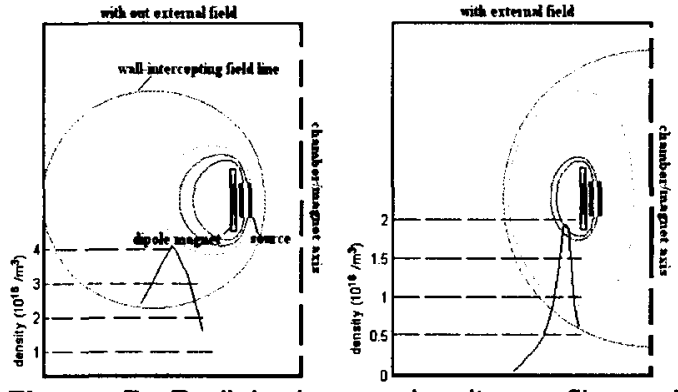

Figure 7. Radial plasma density profile and magnetic field line mapping

The peak equatorial density in the no external field case was $\sim 4 \times 10^{16} \mathrm{~m}^{-3}$. The peak equatorial density with the external field was $\sim 2 \times 10^{16} \mathrm{~m}^{-3}$. The magnetic field in the region of the source is roughly $0.04 \mathrm{~T}$ without the external field, but when the external field is applied this value drops to roughly $0.035 \mathrm{~T}$. The magnetic field pressure in the region of the source therefore drops by $\sim 33 \%$ when the external field is applied. Given that the source $\beta$ may be of order 1 , this change in magnetic field pressure may account for the drop in equatorial density between the two cases; that is to say that the higher $\beta$ of the external field case may have led to more plasma escaping the magnetic field in the source region and a corresponding drop in the plasma measured in the equatorial region.

Estimating the particle and energy inventory of the plasma configuration prior to source shutoff adds further insight to this possibility. Making the course simplifications that the plasma is a uniform density $\sim 10^{16}$ populating a cylindrical shell $10 \mathrm{~cm}$ tall, $5 \mathrm{~cm}$ wide at $\mathrm{R}=18 \mathrm{~cm}$, the resulting plasma volume $\sim$ $.006 \mathrm{~m}^{3}$, and so the total particle inventory $6 \times 10^{13}$. Assuming the $11.6 \mathrm{eV}$ per particle found using the swept Langmuir probes described above, the total energy in the plasma before shutoff $\sim 10^{-4} \mathrm{~J}$. The arc source is known to have deposited a total of $\sim 50 \mathrm{~J}$ into the plasma. Even if $99 \%$ of this energy is lost, the measured total energy is still several orders of magnitude below what is expected from the source energy deposition budget. This would also be explained by large plasma losses due to high local $\beta$ near the source. As will be discussed shortly, the decay rate of the plasma indicates a power loss of $\sim 0.1 \mathrm{~W}$ or less after the source is shut off.

As mentioned previously, the Langmuir probes were radially repositioned during multiple shots, providing a 2D (radial-azmuthal) map of the plasma density in the equator of the magnet 
vs. time. These maps were made for both the external field and no external field cases (Figures 8, 9). Both cases share qualitative similarities. Initially, the plasma configuration is asymmetric, with plasma located only directly in front of the source at a radial distance consistent with the magnetic field line mappings from the source to the equator. Shortly thereafter, the plasma may move radially outward before moving azimuthally around the magnet. The plasma gradually gains a roughly azimuthal symmetry and a radially peaked configuration, although region in front of the source is slightly

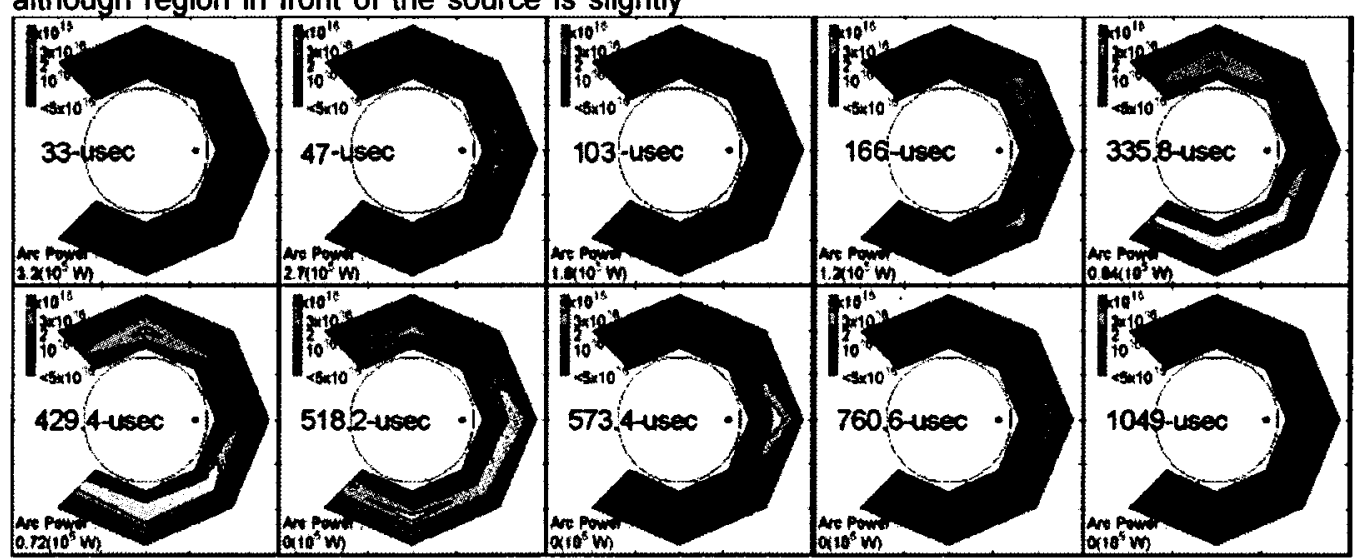

Figure 8: no external field

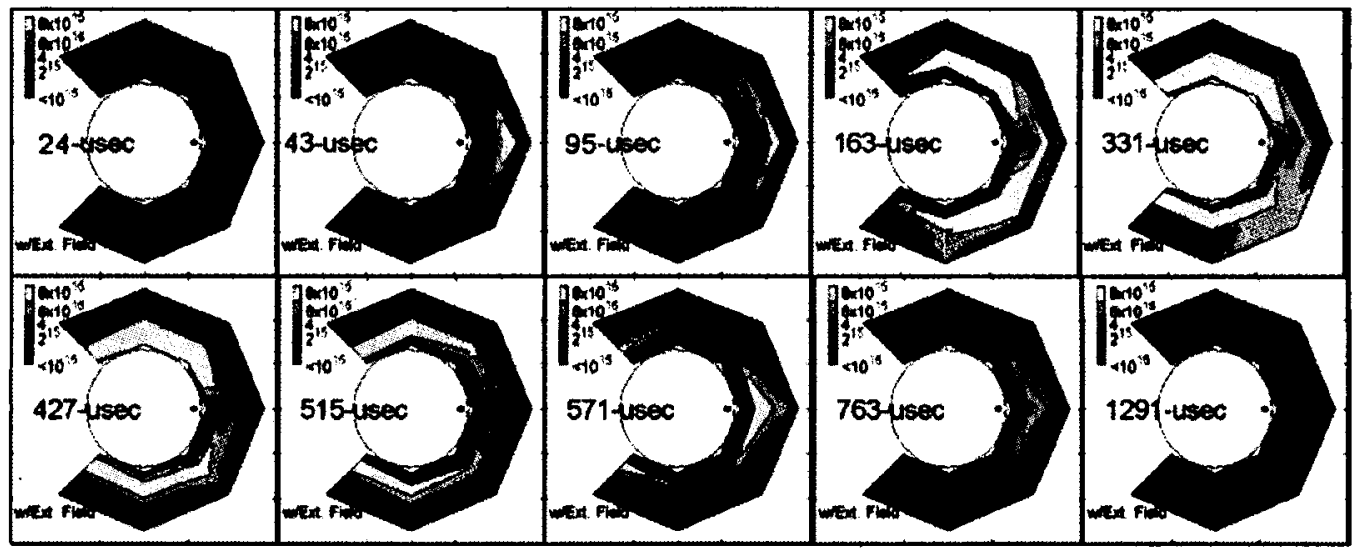

Figure 9: external field

\section{Plasma Decay Rate}

The plasma decay after source shutoff was measured for both the external field and no external field cases (Figure 10). It is seen that the plasma in the no external field case decays faster than in the external field cases, and that the external field case has two distinct decay times. Assuming the density decay is of the form: depleted during plasma production. Once plasma production is shut off, the depleted region transitions to an enhanced region, while the azimuthally symmetric, radially peaked profile remains at other locations. The plasma then gradually decays. The cause of the transitions from asymmetric to quasi-symmetric, and then back to asymmetric configurations are unknown, but may be due to either convection of the initially plasma-loaded flux tube or interactions with the vacuum chamber wall. 
$0.66 \mathrm{msec}$. The observed decay time is thus roughly $10 x$ the Bohm time during the later parts of the decay. The reason for the change in decay time for the external field cases is not known.

Using the $-10^{-4} \mathrm{~J}$ of energy in the plasma configuration derived earlier, and taking a conservative (underestimated) fixed decay time of $1 \mathrm{msec}$ leads to a power loss rate of $0.1 \mathrm{~W}$ after the source is shut off. The fact that the energy loss rate observed after the source is shut off is roughly $0.1 \mathrm{~W}$ compared to the roughly $100 \mathrm{~kW}$ deposited into the plasma by the source during operation may further indicate that plasma/energy loss during source operation is due to conditions in the region of the source.
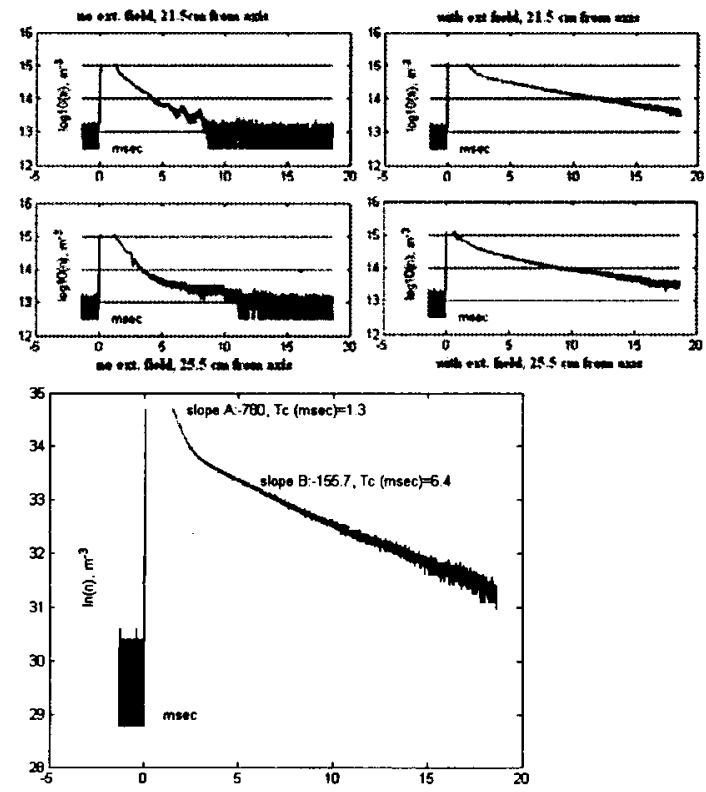

Figure 10: Decay rates

\section{CONCLUSION}

The measurements conducted indicate that the cascaded arc can couple on the order of $100 \mathrm{~kW}$ into the plasma, heating it to roughly $11 \mathrm{eV}$. The characteristic decay time as been measured and is roughly $6 \mathrm{msec}$, an order of magnitude better than the estimated Bohm time. The data also suggest that the plasma produced at the source is high $\beta$. The equatorial plasma density transitions from an initially asymmetric configuration early in the shot to a quasisymmetric configuration during plasma production, and then returns to an asymmetric configuration when the source is shut off. The exact reasons for these changes in configuration are unknown, but convection of the loaded flux tube is suspected.

The next set of experiments, already under development, will reposition the plasma source so that the locally high $\beta$ plasma is directly injected into the equatorial region, as opposed to the present configuration where plasma must flow from the interior of the magnet to the equatorial region.

\section{BIBLIOGRAPHY}

1) Mini-magnetospheric plasma propulsion: Tapping the energy of the solar wind for spacecraft propulsion, R.M. Winglee, J. Slough, T. Ziemba, and A. Goodson, J. Geophys. Res., 10521067 (2000).

2) Parameterization of the laboratory performance of the Mini-Magnetospheric Plasma Propulsion (M2P2) prototype, Ziemba et al., Proc. 2001 International Electric Propulsion Conference.

3) High Beta Plasma for Inflation of a Dipolar Magnetic Field as a Magnetic Sail, by Slough et al., Proc. 2001 International Electric Propulsion Conference.

4) Axial temperatures and electron densities in a flowing cascaded arc: model versus experiment, Beulens, J.J., de Graaf, M.J., Schram, D.C, Plasma Sources Science and Technology, 1993, pg 180-189. 